\title{
AN APPROACH TO 3D DIGITAL MODELING OF SURFACES WITH POOR TEXTURE BY RANGE IMAGING TECHNIQUES. 'SHAPE FROM STEREO' VS. 'SHAPE FROM SILHOUETTE' IN DIGITIZING JORGE OTEIZA'S SCULPTURES
}

\author{
J. García Fernández ${ }^{\mathrm{a}}$, A. Álvaro Tordesillas ${ }^{\mathrm{b}}, \mathrm{S}$. Barba $^{\mathrm{c}}$ \\ ${ }^{a}$ Architectural Photogrammetry Lab., College of Architecture, University of Valladolid - jgarciaf@mtu.edu \\ b Dept. of Graphical Representation, College of Architecture, University of Valladolid - tordesillas@arq.uva.es \\ c Dep. of Civil Engineering, Faculty of Engineering, University of Salerno - sbarba@unisa.it
}

\author{
Commission V, WG V/4
}

KEY WORDS: Cultural Heritage, Modelling, Radiometric-geometric comparison, Surface

\begin{abstract}
:
Despite eminent development of digital range imaging techniques, difficulties persist in the virtualization of objects with poor radiometric information, in other words, objects consisting of homogeneous colours (totally white, black, etc.), repetitive patterns, translucence, or materials with specular reflection. This is the case for much of the Jorge Oteiza's works, particularly in the sculpture collection of the Museo Fundación Jorge Oteiza (Navarra, Spain).

The present study intend to analyse and asses the performance of two digital 3D-modeling methods based on imaging techniques, facing cultural heritage in singular cases, determined by radiometric characteristics as mentioned: Shape from Silhouette and Shape from Stereo. On the other hand, the text proposes the definition of a documentation workflow and presents the results of its application in the collection of sculptures created by Oteiza.
\end{abstract}

\section{INTRODUCTION}

The transitions from analogue to digital photogrammetry, and the rapid development of personal computers processing capacity, have allowed a theoretical-practical framework to develop. This guarantees digital modelling of threedimensional shapes with high levels of accuracy and reliability in terms of geometry and radiometry.

The 3D digital modelling based on digital photogrammetry has become one of the most important tools in solving actual needs for heritage analyses, procedural modelling and accuracy requirement in $3 D$ documentation. Connected with this goal has been conducted the R\&D project: HAR $201^{1}$ Low cost digital survey: sculpture pieces with poor texture by Jorge Oteiza. During the documentation stage, singular pieces of poor textures surfaces presented special difficulties for an accurate $3 \mathrm{D}$ digital reconstruction. These were the cases for much of the Jorge Oteiza's sculptures in the collection of the Museo Fundación Jorge Oteiza (Navarra, Spain). These works are a set of small pieces sculpted in white chalk, white marble, and welded sheet metal that was painted black (Fig. 1). The collection, which Oteiza never gave graphic definition prior to their creation, has not been subjected to analytic processes or metric documentation.

\section{THE MODELLING METHODS}

In order to generate a reliable documentation strategy, the study begins with the theoretical analysis of two methods of digital image-based survey. While having the premise of carried on procedures focused on low cost technologies (based on image information), the selected methods have radically different

\footnotetext{
${ }^{1}$ The project is financed by the MICINN (Spanish Ministry of Science and Innovation).
}

performances in terms of dependence on the object texture: Shape from Silhouette - SfSi, based on the detection of the item shape; and Shape from Stereo - SfS ,based on detection of homologous pixels in stereo/multiple image pairs.

\subsection{Reconstruction based on geometric properties: the method Shape from Silhouette (SfSi)}

One of the major difficulties for 3D reconstruction in cultural heritage is the poverty of information on images to reconstruct the scene. This occurs because, at present, the implemented algorithms in the most common software are based on the massive correspondence of pixels or set of pixels, from a theoretically stereoscopic positioning. The SfSi method, however, bases reconstruction processes on $3 \mathrm{D}$ shape detection from different viewpoints. The idea of using silhouettes for this purpose was first introduced in 1974 by Baumgart and formalized by Laurenti (Laurentini, 1994). This computes intersections of the visual pyramids formed by the silhouettes (generatrix) and the director beam (irregular pyramid axis) from a well-known viewpoint (Haro, 2012). Therefore, this method obtains the surface and the volume contained within the intersection of both visual pyramids, Visual Hull (Fig 2). The visual pyramid can follow two paths, either by an approach based on volume (voxels) or by an approach based on surfaces:

- Approach based on voxels: The space segmentation occurs into a large number of cubes (volumetric elements), called voxels, and the shape is represented on a number of these three-dimensional measurement units. Therefore, the Visual Hull resolution is defined by the size of the voxels and the discrimination ability of full and empty voxels (Forbes, 2007). 


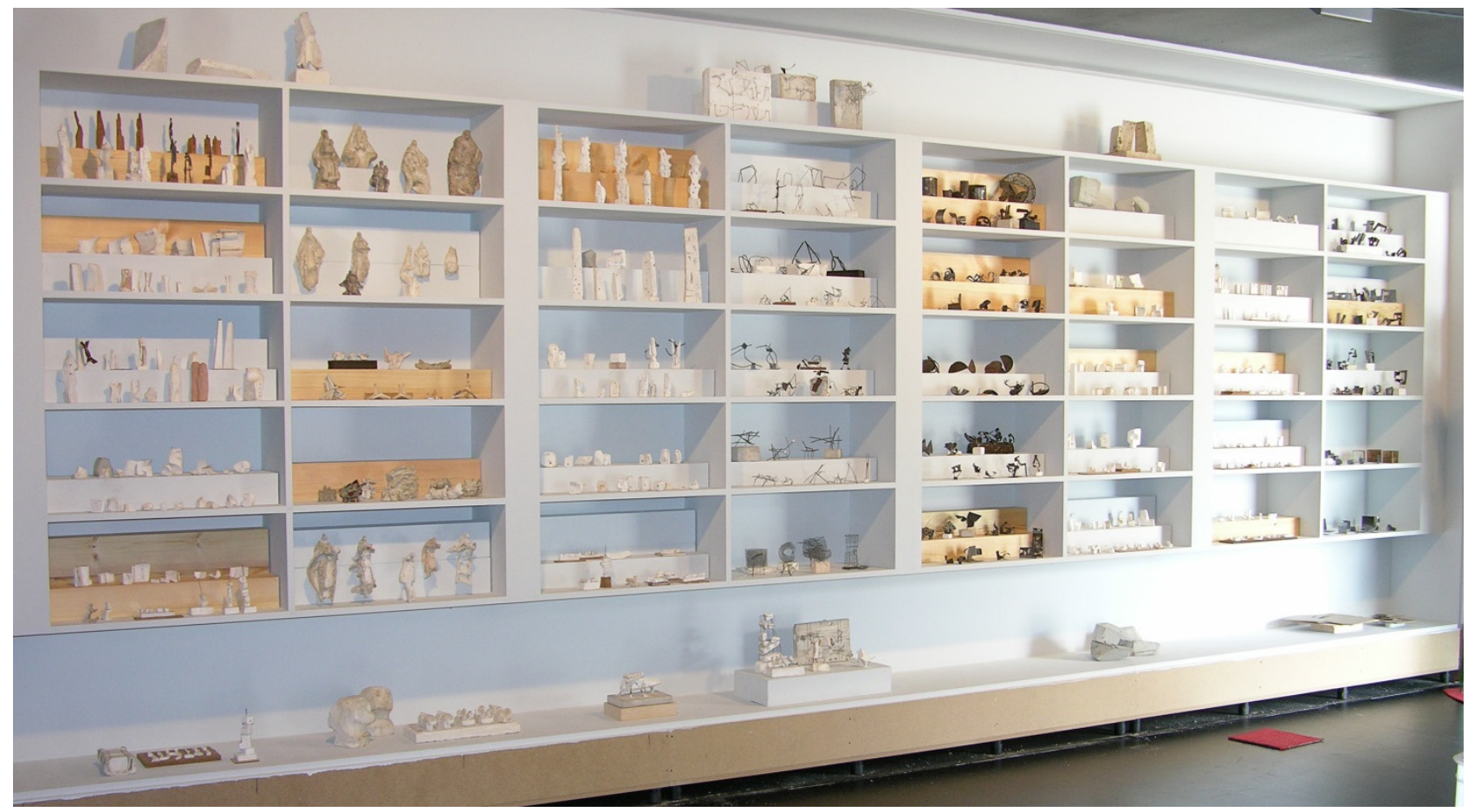

Figure 1. Collection of sculptures by Jorge Oteiza, Museo Fundación Jorge Oteiza, Navarra, Spain

- Approach from surfaces: The second path is defined by the surface analysis. The process begins with the specific geometry analysis of the visual pyramids, and comprises the intersection calculus of polygons. This intersection is defined by the spatial projection of the silhouettes and it is computed by various procedures that prioritize calculation speed (real time processing) or the quality of geometric result (Michoud et al., 2006).

\subsection{Reconstruction based on radiometric properties:} the method Shape from Stereo (SfS)

In the other case, SfS bases acquisition process and 3D reconstruction in the encounter and detection of homologous points (RGB pixels) between stereo and multiple images. Although dense stereo correspondence has been a widely studied problem, the evolution of correspondence and densification algorithms continue to rise. Today, analysing correspondence structures just based on local measurements, such as image intensity (or colour) and phase (Ogale and Aloimonos, 2005), is not enough. We must also consider solutions based on external geometric constrains, symbolic descriptions or patterns (Zhang et al., 2014). Although the study is focused on the SfS method, the results can be extended to the more general Structure from Motion (SfM) method, where internal camera parameters do not need to be known. This project's preference is to conduct digital reconstruction based on the accurate camera calibration (internal orientation), made by the calibration module on PhotoModeler Scanner. However, current developments based on reverse engineering make possible the simultaneously reconstruction of 3D points obtaining (recovering) projection matrices, and maintaining similar accuracies in the correspondence and massification of points.
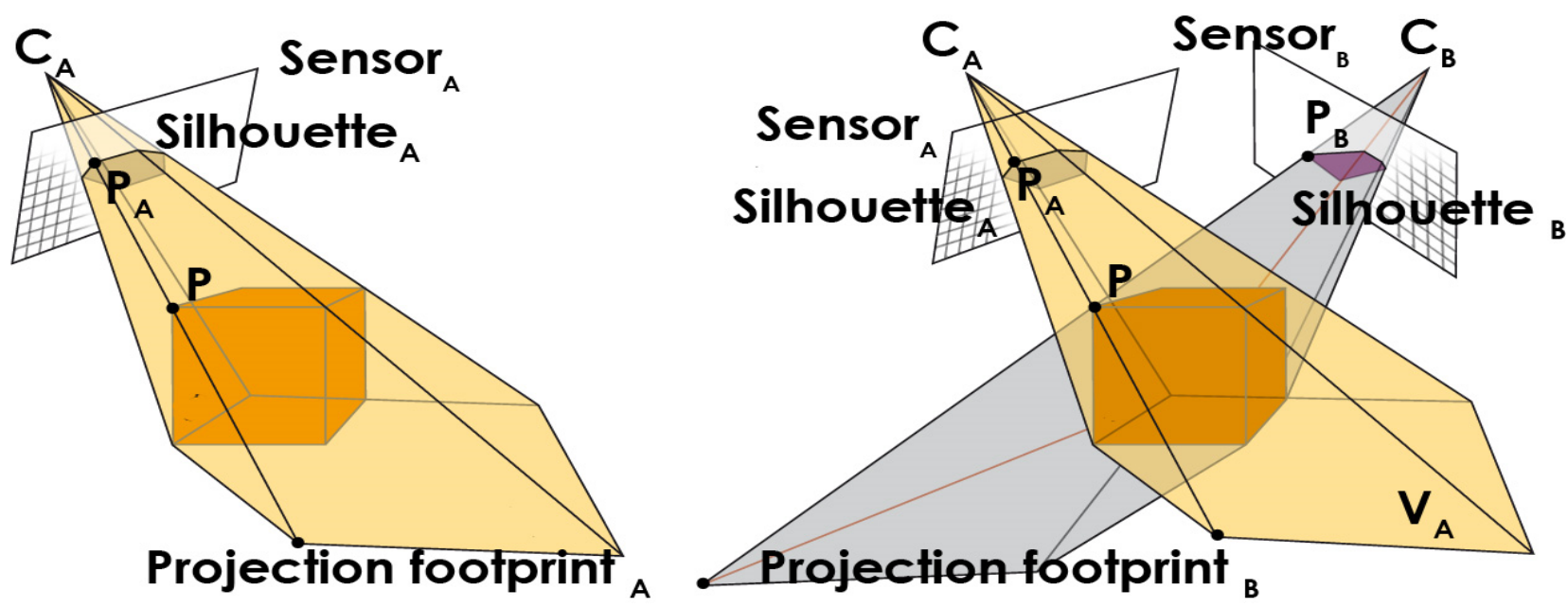

Figure 2. Left: visual cone from a single point of view. Right: intersection of multiple cones on a three dimensional object 


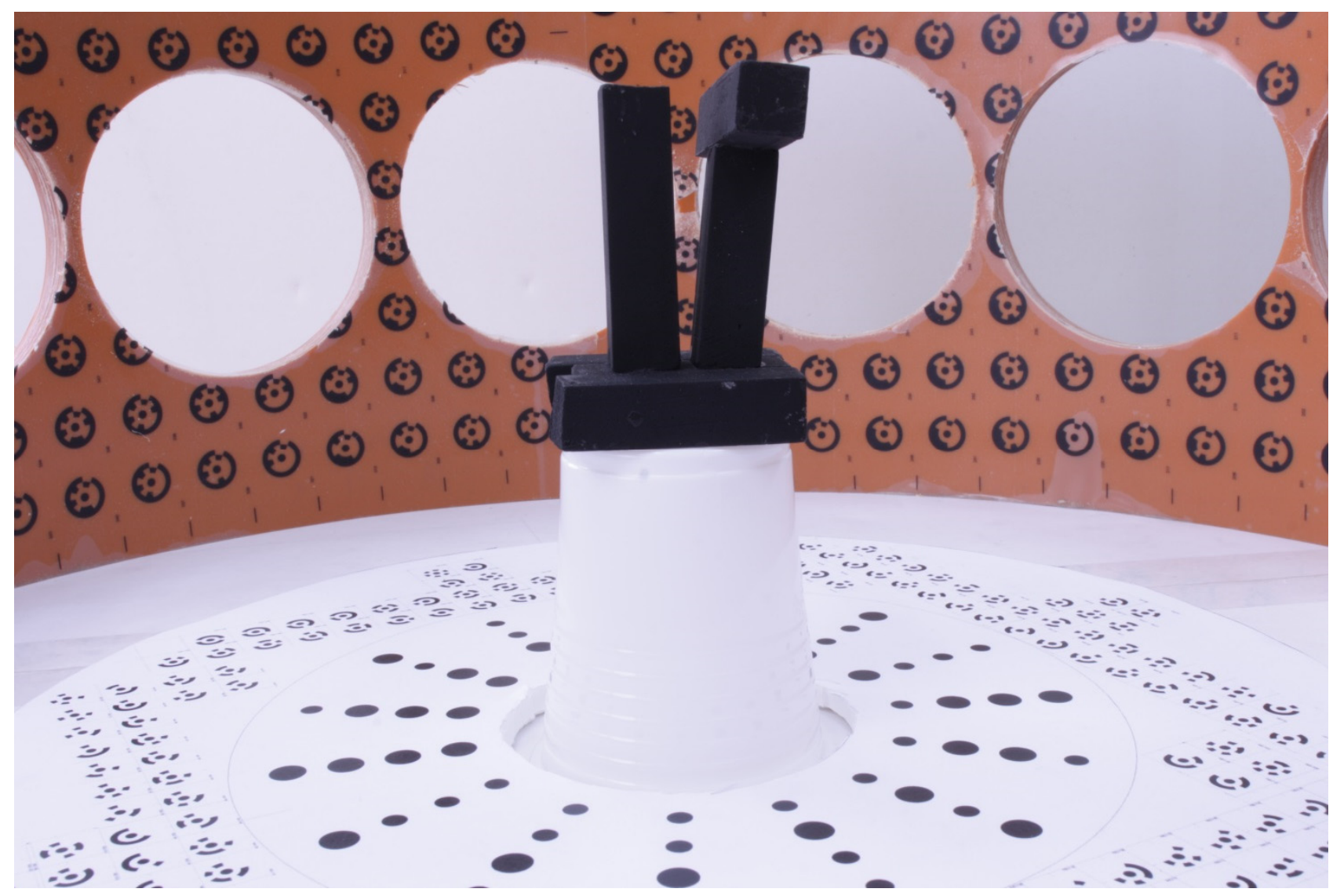

Figure 3. Barrel of Capture, designed for conducting simultaneous documentation process based on SfSi and SfS

\subsection{Documentation process}

The need for capturing images while maintaining optimal (and similar) external conditions, forced us to establish a capture flow in parallel. Once the strengths and limitations of the two methods become known, it was designed a controlled space named the Barrel of Capture (Fig. 3). This Barrel, consisting in a rotary mechanized structure, was designed to supply the optimal capture condition of both methods. To accomplish this, were first established criteria for ideal 3D reconstruction under the typical conditions for each technique. That includes conditions for image capture, taking into account that the data processing was conducted with PhotoModeler Scanner 1.0.4 (for SfS Method) and CX2 Strata 3D Photo v2.1 (for SfSi method, Fig. 1). Therefore, the barrel had to meet the following conditions:

\section{(for SfS method)}

- Permissibility of photo capture in a parallel or close to parallel orientation. Cameras with relation base/height of $1 / 4$. Images with an overlap close to $40 \%$;

- space in the scene for identifying code targets of the reconstruction software (PhotoModeler Scanner);

- scene structure capable to document the piece sample while occupying the entire frame of the sensor;

- scenario that allows the entry of uniform light, without shadows.

\section{(for SfSi method)}

- Permissibility steps capture every 24 degrees to the lower ring and 40 degrees to the upper ring;
- space in the scene for identifying templates of the reconstruction software (Strata 3D Photo);

- scenario with the possibility of swapping templates. Templates with different colours were used in order to avoid colour contained in the scene.

For the metric comparison was selected a chalk sculpture with homogeneous colour and dimensions of $120 \mathrm{~mm} \times 8 \mathrm{~mm} \times 62 \mathrm{~mm}$. The documentation process, applied to the selected sample, led us to the creation of two digital 3D models (one model for each method). Figure 4 shows the relative orientation of the cameras and the mesh obtained from SfSi method.

\section{DATA COMPARISON}

Once established the theoretical framework and the procedures used for a parallel documentation, this study focuses on the metric comparison of digital models obtained from both techniques in order to assess the potentials in the accurate modelling, as well as the limitations. For an accurate comparison, a geometric reference with a rigorous reliability was necessary. In the case of studied sculptural pieces, the lack of earlier dependable documentation led to the surface modelling by using High Dynamic Range Scanning (HDRS) Technique (Zhang and Yau, 2009). For this purpose, this study used the scanner Perceptron ScanWorks V5 with an articulated arm Romer. Because the starting position of the laser beam (beam pattern) and the arrival through two CCD sensors (in the case of the Perceptron V5) are known, it is possible to calculate the incidence angle of the beam plane relative to the documented surface. 

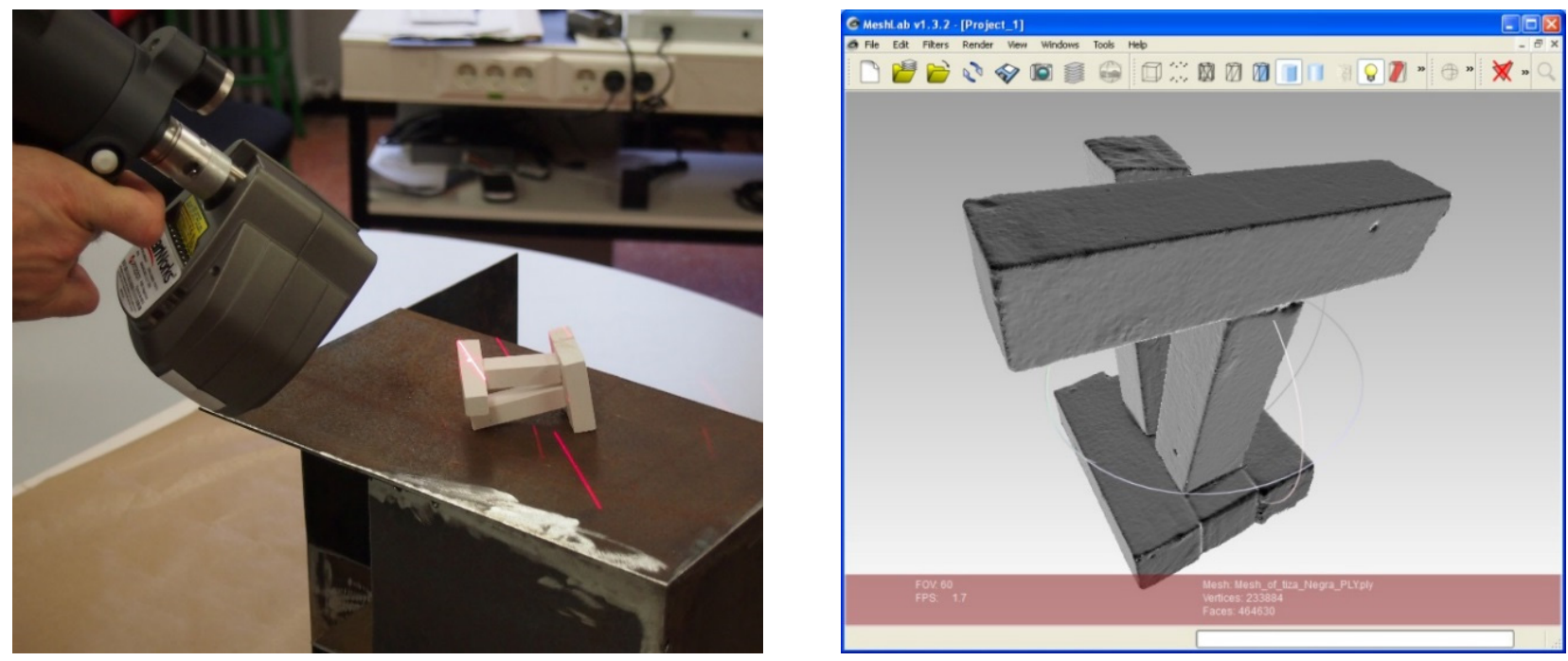

Figure 5: Left: laser beam plane projected onto a piece for three-dimensional reconstruction. Right: mesh obtained from 3D point cloud.

Using two sensors, each capturing half of the projected plane, enhances the accuracy of the feature being documented and maintains small dimensions of the device base.

The scans are referenced to a unique (and portable) coordinate system through the articulated arm. The HDRS enables calibration of the emitted bean intensity with respect to surface properties of the documented object. This allows reconstruction of surfaces and materials with different properties - semitranslucent materials (high beam penetration), dark materials (high light absorption), or highly reflective materials (specular) - fitted to those required in this project (Fig. 5).

To perform the metric comparison, the proposed project used Innovmetric Polyworks software and two modules. First, IMAlign creates surface based on the laser scanner point cloud, without application of smoothing, tolerance or reduction. Second IMInspect aligns data and calculates the metric differences between the reference and the surfaces obtained from both SfSt and SfSi methods (Fig. 6, A-B). The conducted comparison used the criteria of shortest distance perpendicular to the plane. Therefore, each point was correlated with an error vector (Fig. 6, C). The result of the comparison are shown in Table 1.

\section{COMPARISSON ANALYSIS AND APPLICATION}

The behaviour of the main parameters (Tab. 1) reflects how insofar as homologous points and its reduced neighbourhood cannot be identified, the method $\mathrm{SfSi}$ will have better performances compared to the SfS method. As described in the data comparison, the SfSi method directed the $3 \mathrm{D}$ reconstruction of the entire surface without jumping or discontinuities (Gig. 6 $\mathrm{B}, \mathrm{C})$, while keeping the error in range below $3 \mathrm{~mm}$.

The metric comparison, together with the previous performance analysis, allows us to define SfSi the more efficient method for the lower variance and for the richness of the radiometric information: in this line was defined a systematic survey of digital 3D sculptures with poor radiometric information, based on the method SfSi.

Its use will have better results while documentation criteria mentioned in section 2.3 are fulfilled and the expected accuracy is within the range. The application of the SfSi methods in the Jorge Oteiza collection (Museo Fundación, Navarra) able possible the creation of an interactive and online digital database of the not documented pieces (Fig. 7).
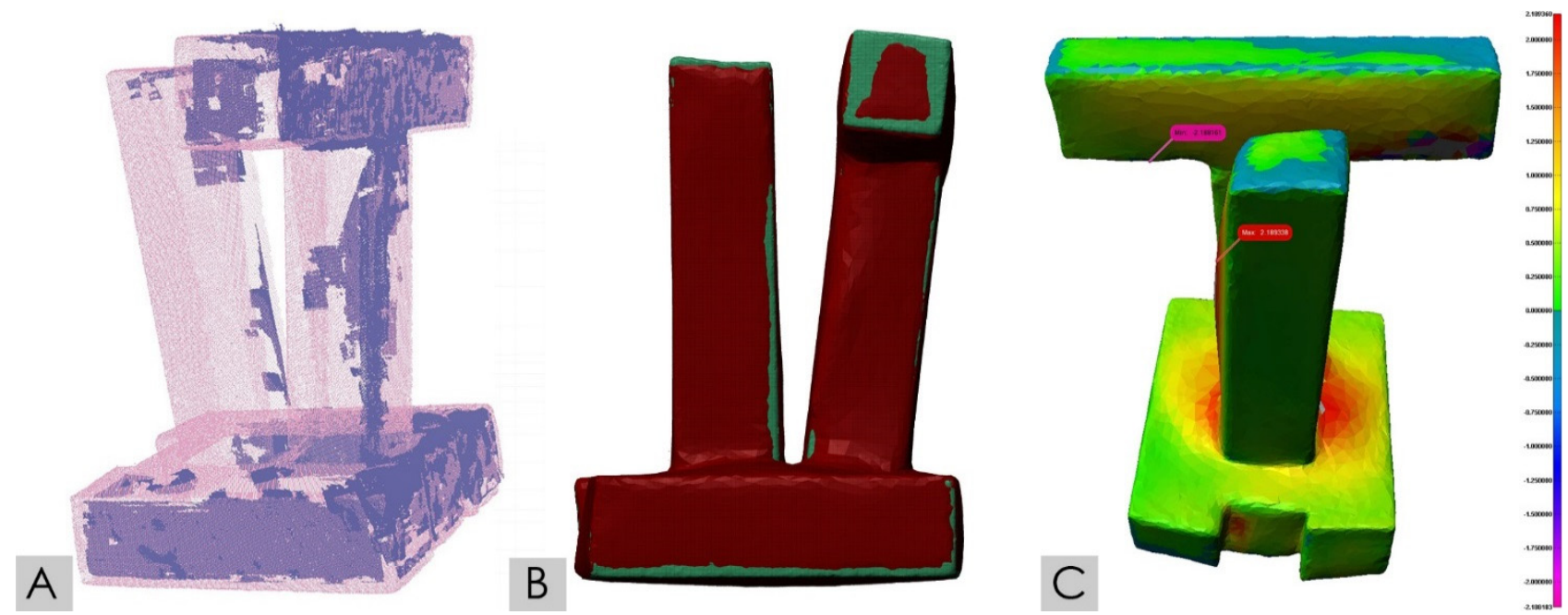

Figure 6. A: overlapping point clouds obtained from Shape from Stereo method (darker) versus laser scanner data (lighter). B: overlapping meshes obtained from Shape from Silhouette (in red) versus laser scanner data mesh (in green). C: error scale map 
Table 1. Main parameters in the comparison process

\begin{tabular}{|c|c|c|}
\hline Parameter & SfSi (vs Reference) & SfS (vs Reference) \\
\hline Point total & 10344 & $9174(96.40 \%)$ \\
\hline Points in the range of $\pm 1.5 \mathrm{~mm}$ & $7100(68.64 \%)$ & $9432(99.12 \%)$ \\
\hline Points in the range of $\pm 2.5 \mathrm{~mm}$ & $9852(95.24 \%)$ & $0.438 \mathrm{~mm}$ \\
\hline Point distribution & Homogeneous & $0.3278 \mathrm{~mm}$ \\
\hline Error average & $1.3474 \mathrm{~mm}$ & $0.5174 \mathrm{~mm}$ \\
\hline Standard Deviation & & \\
\hline
\end{tabular}
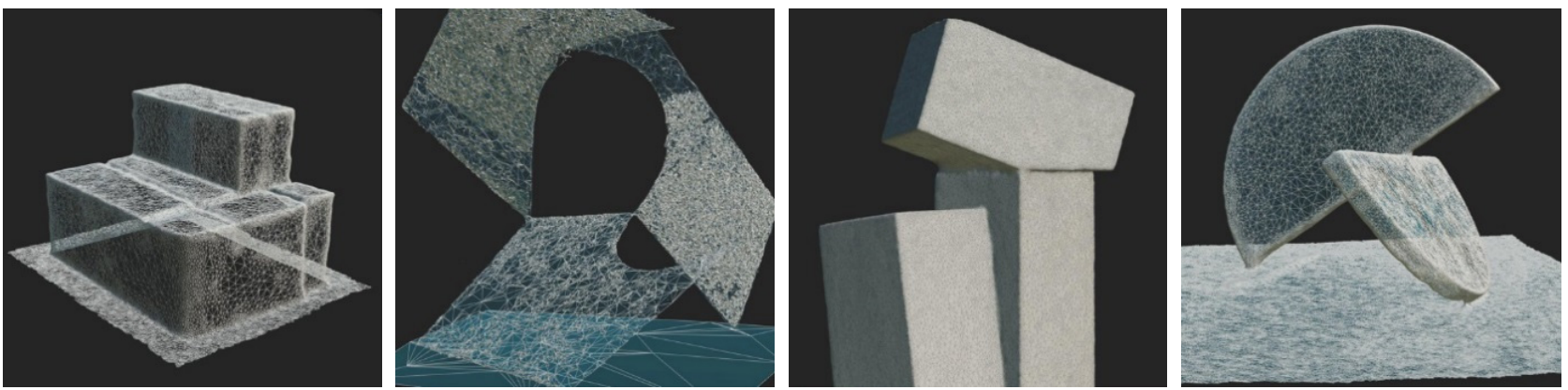

Figure 7. Database of digitized Oteiza's sculptures based on the proposed workflow

\section{CONCLUSIONS AND FUTURE WORKS}

The present text, allows the estimation of real potentialities and limitations of two range imaging techniques: SfSi and SfS, applied in the field of cultural heritage. The study bases this estimation in the analysis of geometric modelling behaviour and the geometric comparison of 3D models. The obtained result helps to improve the selection and use of these methods in the cultural interpretation, dissemination and preservation. In particular, the study of the Shape from Silhouette method allows us to retake math implementations over 20 years ago, which can be reused now thanks to the new technologies and new cultural needs. Related to the documentation of sculptural pieces with of poor texture (dark colours, repetitive patterns and materials with little response to light stimuli); this method obtains significant advantages over others based on range information or massive correspondence of pixels. Its gain will be higher while the texture of the piece is more homogeneous. The need to improve accuracy while is efficiently reconstructed the geometry of shapes with poor texture compels us to continue the study in two new research lines. On one hand, it is the analysis of the influence and behaviour of visual hull calculus in samples characterized by both curves and straight edges; in the other hand, it is the analysis of geometric reconstruction based on a unified system that comprises both SfSi and SfS.

\section{ACKNOWLEDGE}

We gratefully acknowledge support from the Laboratory of Architectural Photogrammetry (University of Valladolid, Spain), and from the Museo-Fundación Jorge Oteiza in Pamplona (Spain).

\section{REFERENCES}

Forbes, K., 2007, Calibration, Recognition, and Shape from Silhouettes of Stones, PhD Thesis, University of Cape Town.

Haro, G., 2012, Shape from Silhouette Consensus. Pattern Recognition, 45(9), pp. 3231-3244.

Laurentini, A., 1994, The visual hull concept for silhouettebased image understanding. IEEE Trans, Pattern Analysis and Machine Intelligence, 16(2), pp. 150-162.

Michoud, B., Guillou, E. and Bouakaz, S., 2006, Shape From Silhouette: Towards a Solution for Partial Visibility Problem. Eurographics, pp. 13-16.

Ogale, A. and Aloimonos, Y., 2005, Shape and the stereo correspondence problem. International Journal of Computer Vision, 65(3), pp. 147-162.

Zhang, S. and Yau, S., 2009, High Dynamic Range Scanning Technique. Optical Engineering Journal, 48(3), 033604.

Zhang, D., Lee, D. and Chang Y., 2014, A New Profile Shape Matching Stereovision Algorithm for Real-time Human Pose and Hand Gesture Recognition. International Journal of Advanced Robotic Systems, 11(16), pp. 1-12. 